\title{
Reemplazo renal en el post-operatorio de niños sometidos a cirugía cardíaca con circulación extracorpórea
}

\author{
PILAR OVALLE B. ${ }^{1}$, ANDREA VOGEL S. ${ }^{2}$, GUILIANA CÓRDOVA L. ${ }^{3}$, \\ JAIME CERDA L. ${ }^{4}$, FELIPE CAVAGNARO SM. ${ }^{5}$ \\ 1. Pediatra, División de Pediatría, Pontificia Universidad Católica de Chile. \\ 2. Nefrólogo Pediatra, División de Pediatría, Pontificia Universidad Católica de Chile. \\ 3. Intensivista Pediatra, División de Pediatría, Pontificia Universidad Católica de Chile. \\ 4. Pediatra Especialista en Salud Pública, Departamento de Salud Pública, Pontificia Universidad Católica de Chile. \\ 5. Nefrólogo Pediatra, Clínica Alemana-Universidad del Desarrollo.
}

\begin{abstract}
Renal replacement therapy after cardiac surgery with extracorporeal circulation
\end{abstract}

Objective: Acute kidney injury (AKI) is a frequent complication of cardiac surgery with cardiopulmonary bypass (CPB). The aim of this study was to determine the incidence of renal replacement therapy (RRT) requirement in children undergoing surgical repair for congenital cardiopathies and identify the risk factors for requiring RRT. Patients and Methods: A retrospective, descriptive study was performed. The records of patients under 18 years undergoing cardiac surgery with CPB (January 2001 to July 2008) who required RRT $(n=15)$ and from a random patients sample that did not require it $(n=57)$ were analysed. Results: Fifteen children out of 942 required acute RRT (1.6\%). Initial RRT was peritoneal dialysis (PD) in all of them. The main indications were hypervolemia and/or oligoanuria. Ten patients experienced complications associated to therapy and 14 patients had a history of serious intraoperative events. In multivariated analysis, hypotension (OR 36.0, CI 95\%: 5.5-235.6) and serious low cardiac output (LCO) (OR 14.1, CI 95\%: 1.47-135.32) were significant risk factors for RRT requirement. In turn, the presence of intraoperative events was significantly associated with the occurrence of hypotension (OR 28.9, CI 95\%: 3.6-233.9) and LCO (OR 5.3, CI 95\%: 1.126.6) during the post-surgical evolution. Conclusion: In this experience, severe hypotension and low cardiac output were significant risk factors for RRT requirement. The incidence of RRT was $1.6 \%$. Renal function should be closely evaluated in those patients with surgical events. RRT should be early considered in those patients with risk factors for renal failure.

(Key words: Acute kidney failure, cardiac surgical procedures, cardiopulmonary bypass, congenital heart defects, peritoneal dialysis, renal replacement therapy).

Rev Chil Pediatr 2012; 83 (1): 24-32

Trabajo recibido el 09 de enero 2011, devuelto para corregir el 14 de marzo de 2011, segunda versión el 20 de junio de 2011 , aceptado para publicación el 22 de noviembre de 2011.

Correspondencia a:

María del Pilar Ovalle Besa

E-mail: pilarovalleb@gmail.com 


\section{RESUMEN}

Introducción: La insuficiencia renal aguda es una complicación frecuente de la cirugía cardíaca con circulación extracorpórea (CEC). Objetivo: Determinar la incidencia de requerimiento de terapia de reemplazo renal (TRR) en niños sometidos a reparación quirúrgica de cardiopatías congénitas, caracterizarlos e identificar factores de riesgo de requerir TRR. Pacientes y Método: Se realizó un estudio retrospectivo descriptivo con un componente analítico. Se revisaron las fichas clínicas de los pacientes menores de 18 años sometidos a cirugía cardíaca con CEC (enero 2001 a julio 2008) que requirieron TRR $(n=15)$ y de una muestra aleatoria de pacientes que no la requirieron $(n=57)$. Resultados: De los 942 niños operados, 15 requirieron TRR aguda (1,6\%). En todos, la TRR inicial fue peritoneodiálisis (PD). Las principales indicaciones fueron hipervolemia y/o oligoanuria. Diez pacientes presentaron complicaciones asociadas a la terapia y 14 tenían antecedentes de incidentes intraoperatorios graves. En forma multivariada, la hipotensión arterial (OR 36,0 IC 95\%: 5,5-235,6) y el hipodébito grave (OR 14,1 IC 95\%: 1,47-135,32) fueron factores de riesgo significativos de requerir TRR. A su vez, la presencia de incidentes intraoperatorios se asoció significativamente al desarrollo de hipotensión (OR 28,9 IC 95\%: 3,6-233,9) e hipodébito (OR 5,3 IC 95\%: 1,1-26,6) durante la evolución post-quirúrgica. (Palabras clave: Insuficiencia renal aguda, cirugía cardíaca, circulación extracorpórea, cardiopatías congénitas, peritoneodiálisis, terapia de reemplazo renal).

Rev Chil Pediatr 2012; 83 (1): 24-32

\section{Introducción}

La insuficiencia renal aguda (IRA) es una complicación grave en niños hospitalizados en Unidades de Paciente Crítico Pediátricas (UCIP) y su incidencia va desde 2,5 a $4,5 \%$ según estudios prospectivos ${ }^{1,2}$. Esta patología condiciona un incremento importante de la morbimortalidad, llegando a cifras de mortalidad de 27,3\% comparado con 2,4\% en pacientes sin IRA ${ }^{1}$. Dentro de las patologías asociadas, la reparación quirúrgica de cardiopatías congénitas con uso de circulación extracorpórea (CEC) es una de las principales causas de IRA en las UCIP, con una incidencia reportada en distintas series entre 17 y $72 \% \%^{2-5}$. La amplitud de este rango puede deberse, entre otros factores, a la variedad de definiciones de IRA utilizadas y a la diversa complejidad de las cirugías incluidas. El uso de CEC per se condiciona una serie de factores implicados en el deterioro de la función renal ${ }^{4,6}$. Durante la evolución post-operatoria de estos pacientes, la necesidad de terapia de reemplazo renal aguda (TRR) oscila, según distintos estudios, entre 2,9 y $17 \% \%^{3,4,6-10}$. Esta diferencia está dada, principalmente, por los grupos de pacientes evaluados y los criterios de reemplazo empleados.

El objetivo primario de este estudio es de- terminar la incidencia de TRR aguda en los pacientes menores de 18 años durante el postoperatorio de cirugías cardíacas con CEC en el Hospital Clínico de la Universidad Católica de Chile entre enero de 2001 y julio de 2008. Los objetivos secundarios son: describir el grupo de pacientes que requirió TRR durante su evolución post-quirúrgica y las complicaciones asociadas a la terapia e identificar factores pre, intra y post-operatorios asociados a un aumento del riesgo de requerir esta TRR.

\section{Pacientes y Método}

Estudio retrospectivo descriptivo con un componente analítico, en dos fases. En la primera, en base a los registros de la UCIP del Hospital Clínico UC, se revisaron todas las fichas clínicas de los pacientes menores de 18 años que requirieron TRR durante su evolución post-operatoria de reparación quirúrgica de cardiopatías congénitas con uso de CEC entre enero de 2001 y julio de 2008. Se excluyeron los pacientes con TRR previa a la cirugía.

Se registraron los siguientes datos: antropometría, edad, tipo de cardiopatía, cianosis preoperatoria, patologías asociadas, parámetros intra-operatorios (tiempo de CEC, clampeo aórtico, paro cardiocirculatorio y temperatura 
mínima intra-CEC), incidentes intraoperatorios, necesidad de reingreso a CEC y/o de una nueva cirugía, uso de diuréticos y drogas potencialmente nefrotóxicas previo al inicio de la TRR; indicación, tipo, duración y causa de término de la TRR, complicaciones del procedimiento, intervalo entre la cirugía y el inicio de la TRR, tiempo de estadía en UCIP, parámetros de laboratorio (Hematocrito (HTO) post-CEC, creatininemia preoperatoria y al alta, recuento de plaquetas, lactacidemia), número de drogas vasoactivas (DVA) administradas, presión venosa central (PVC), presencia de coagulación intravascular diseminada (CID), hipotensión, hipodébito e hipoxemia grave, complicaciones y causa de muerte.

Se consideró hipervolemia la presencia de edema y PVC elevada en ausencia de falla ventricular; oligoanuria como diuresis $<0,5$ $\mathrm{ml} / \mathrm{kg} / \mathrm{h}$ en $24 \mathrm{hrs}$; hiperkalemia, potasio (K) > $6 \mathrm{mEq} / \mathrm{L}$ que no responde a medidas farmacológicas (bicarbonato de sodio, resinas de intercambio iónico, nebulizaciones con beta- 2 agonistas, furosemida); uremia, nitrógeno ureico sanguíneo (BUN) > 100 mg/dL.

Los eventos clínicos se definieron como sigue: hipotensión arterial persistente: presión arterial (PA) persistentemente $<2$ DE para la edad ${ }^{11}$, que requirió infusión de fluidos y DVA; hipoxemia: saturación arterial de oxígeno (u oximetría de pulso) $<75 \%$ en forma persistente; síndrome de hipodébito: presencia de signos de hipoperfusión clínicos y de laboratorio mantenidos (llene capilar disminuido, oligoanuria, aumento del ácido láctico en sangre $>2 \mathrm{mmol} / \mathrm{L}$, acidosis metabólica (exceso de base $<-4$ ), requerimiento de altas dosis de DVA para mantener una perfusión adecuada). La presencia de CID se determinó por fibrinógeno $<100 \mathrm{mEq} / \mathrm{L}$.

La función renal se determinó como clearence de creatinina según la fórmula de Schwartz ${ }^{12}$, excepto en los recién nacidos de pretérmino, en quienes se consideró como referencia de normalidad el valor de creatininemia esperado según la edad gestacional y los días de vida ${ }^{13}$. No se utilizó la duplicación del valor de creatinina preoperatoria ya que no todos los pacientes tenían una medición previa a la cirugía.
En la segunda fase del estudio, se revisaron los registros clínicos de una muestra aleatoria $(\mathrm{n}=57)$ de pacientes menores de 18 años hospitalizados en la UCIP del Hospital Clínico UC durante el post-operatorio de la reparación quirúrgica de cardiopatías congénitas con uso de CEC, que no requirieron TRR, entre enero de 2001 y julio de 2008. Se obtuvieron los mismos datos señalados anteriormente. Se analizaron 57 registros para obtener una proporción paciente/control entre 1:3 y 1:4. La extracción de los pacientes se hizo con una randomización aleatoria realizada por un programa computacional. Cinco registros clínicos no estuvieron disponibles y fueron reemplazados por una nueva randomización aleatoria, con el mismo programa computacional.

Se realizó un análisis univariado con test no paramétrico (Test de Mann-Whitney y calculo de odds ratio e intervalo de confianza 95\%) para determinar las variables asociadas a la necesidad de TRR. Las variables estudiadas se escogieron en base a la revisión de la literatura. Las variables continuas se expresaron como promedio y desviación estándar (DE) y las categoricas como porcentajes. Luego se efectuó un análisis de regresión logística multivariado para determinar los factores de riesgo de requerir TRR en este tipo de pacientes. En este modelo se incluyeron las variables previas al inicio de la TRR que habían sido significativas en el análisis univariado. Se usó el Programa Computacional SPSS versión 15.

Este estudio fue aprobado por el Comité de Ética del Hospital Clínico de la Pontificia Universidad Católica de Chile.

\section{Resultados}

Entre enero de 2001 y julio de 2008, fueron sometidos a reparación quirúrgica de cardiopatías congénitas con CEC 942 pacientes menores de 18 años. De éstos, 15 evolucionaron con IRA que requirió algún tipo de TRR aguda $(1,6 \%)$. En la tabla 1 se muestran las características de este grupo de pacientes, las indicaciones de TRR, las complicaciones asociadas a la terapia y sus causas de término. Siete pacientes (7/15) eran hombres. El promedio de 


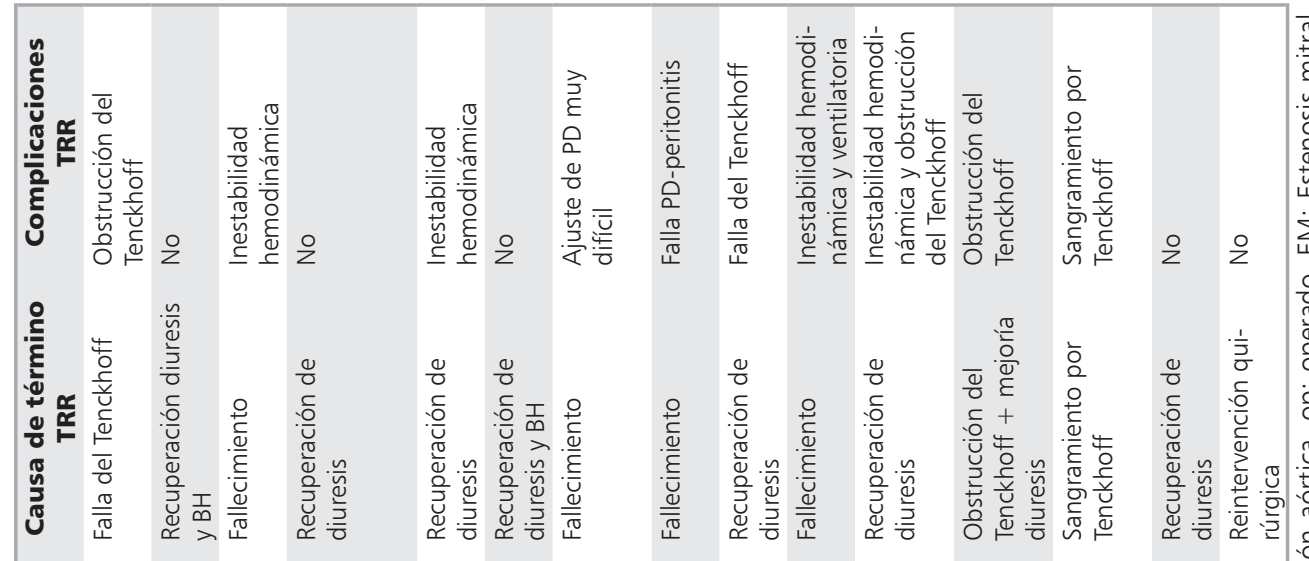

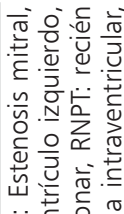

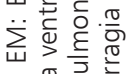

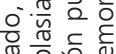

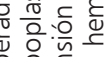

응.을

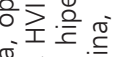

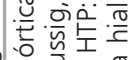

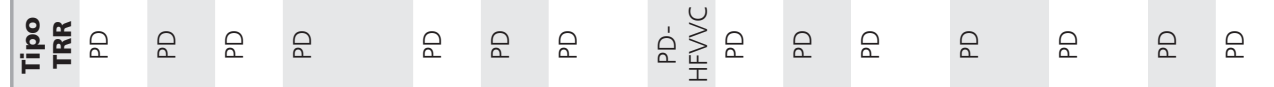

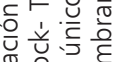

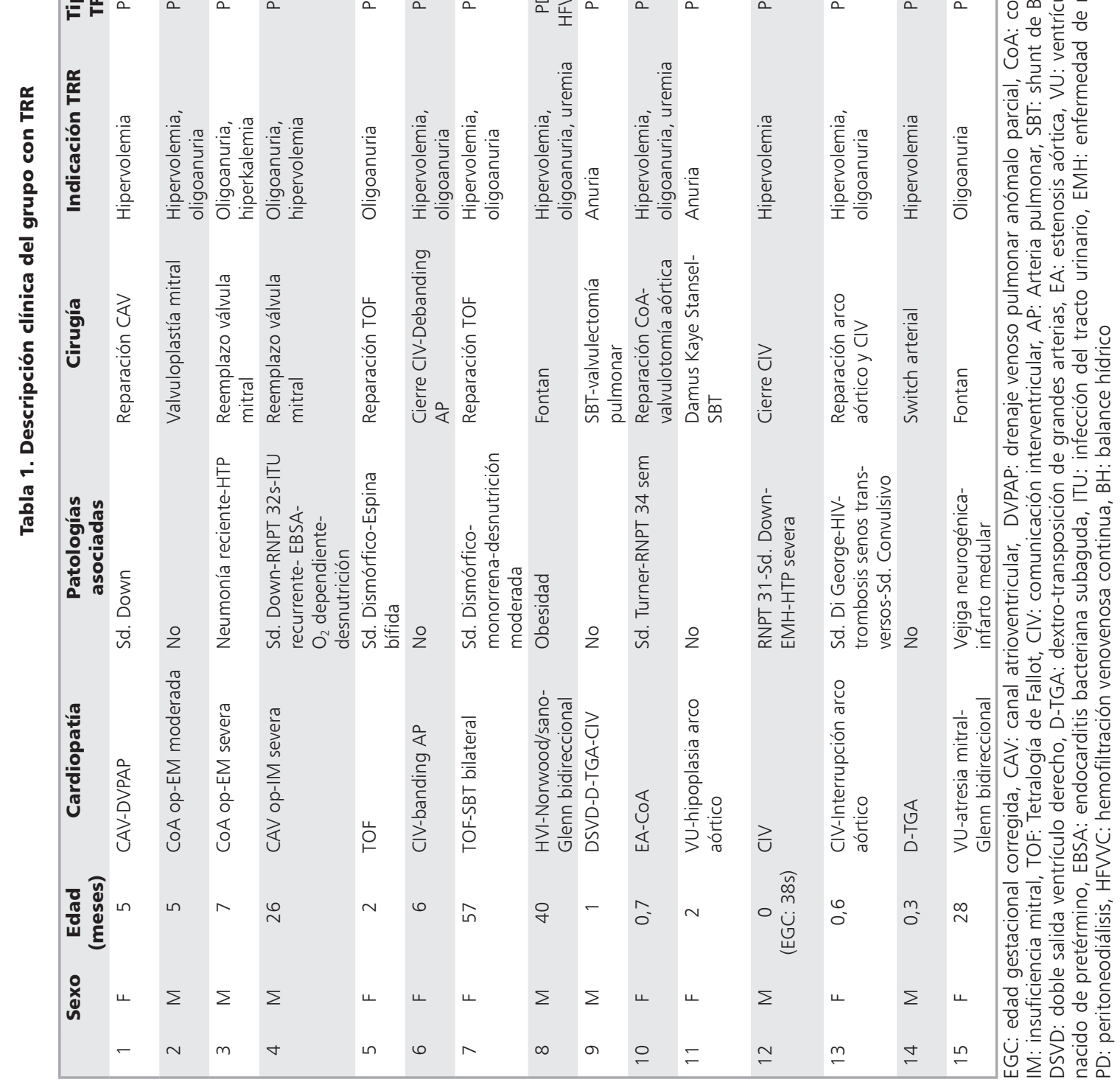


edad fue 12 meses (rango: recién nacido a 4 años 8 meses). Diez pacientes tenían patologías asociadas, destacando entre ellos un paciente monorreno, otro con vejiga neurogénica y otro con antecedente de infección urinaria a repetición. La función renal pre-operatoria fue anormal en 4 de los 10 pacientes en que estaba disponible; 2 de ellos tenían una disminución leve de la filtración glomerular y los otros 2, moderada.

Todos los pacientes recibieron diuréticos previo al inicio de la TRR (furosemida, ya sea en forma exclusiva o en combinación con espironolactona, metolazona o clorotiazida) y 11/15 pacientes recibieron al menos una droga potencialmente nefrotóxica: antinflamatorios no esteroidales, medio de contraste yodado, vancomicina y/o aminoglicósidos. 13/15 niños presentaron hipodébito importante, requiriendo en promedio 4,3 DVA durante su evolución (rango: 3-5 DVA).

Las principales indicaciones de TRR fueron hipervolemia y/o oligoanuria. La uremia se consideró indicación de TRR sólo en aquellos pacientes en que el aumento del BUN influyó en la decisión de iniciar el reemplazo renal debido a la toxicidad secundaria. El tiempo promedio entre la cirugía y la instauración de la TRR fue 97,3 horas (rango: 11-336 hrs). En todos los niños, la TRR inicial fue diálisis peritoneal (PD); en uno de ellos se cambió posteriormente a hemofiltración venovenosa continua (HFVVC) por fracaso de la primera terapia. La duración promedio de la TRR fue 113,2 hrs (rango: 1-601 hrs).

En la tabla 2 se muestran las variables intraoperatorias. En 2 pacientes no hubo clampeo aórtico y sólo en 7 se realizó paro circulatorio. Catorce pacientes (93\%) presentaron incidentes intraoperatorios: sangramiento profuso, bloqueo atrioventricular, falla cardíaca, hipoxia grave, isquemia miocárdica o arritmias. Cuatro pacientes requirieron entrar nuevamente en CEC durante la cirugía y dos debieron ser intervenidos por segunda vez en el post-operatorio inmediato.

Seis pacientes fallecieron, cuatro de ellos durante el período con TRR. Las causas de mortalidad fueron: falla orgánica múltiple (FOM) (2), hemorragia pulmonar (2) y falla cardíaca/hemodinámica grave (2). El prome- dio de días de estadía post-operatoria en la UCIP fue 20,1 días (rango: 2-64 días). Dos niños fueron trasladados a otros centros.

Hubo dos pacientes con indicación de TRR en los que ésta no llegó a realizarse. En uno de ellos, el catéter de PD no funcionó y se logró mejorar la diuresis con furosemida en infusión continua; el otro falleció inmediatamente antes del inicio de la PD.

De los pacientes en que se midió la función renal previo al alta, uno presentaba una disfunción severa (no se conoce su situación preoperatoria) y tres, una disminución de la filtración glomerular moderada (en uno de ellos similar al valor preoperatorio).

Se realizó un análisis univariado para identificar las variables asociadas a un mayor riesgo de requerir TRR. No hubo asociación significativa con edad, peso, patologías concomitantes (tanto en número como en gravedad), cianosis preoperatoria, uso de nefrotóxicos, hipoxemia, función renal preoperatoria ni HTO post-operatorio. Nueve variables se asociaron significativamente a un mayor riesgo de requerir TRR aguda ( $<00$,05) (tablas 2 y 3).

En forma multivariada, la hipotensión grave y el hipodébito persistente se asociaron significativamente a un mayor riesgo de requerir TRR aguda (figura 1). Los OR obtenidos fueron: hipotensión grave: 36 (IC 95\%: 5,513-235,6) e hipodébito persistente: 14 (IC 95\%: 1,5-135,5). Dada la gran influencia que tuvieron estos dos parámetros en el desarrollo de falla renal, se buscaron las variables asociadas a un mayor riesgo de desarrollar hipodébito e hipotensión, encontrándose que los incidentes intraoperatorios se asociaron significativamente a estos parámetros en análisis univariado (figura 2). La bondad de ajuste del modelo multivariado fue $68,3 \%\left(\mathrm{R}^{2}\right.$ Nagelkerke).

\section{Discusión}

Nuestra incidencia de TRR durante la evolución post-operatoria de la reparación quirúrgica de cardiopatías congénitas con CEC en pacientes menores de 18 años fue menor a la reportada en otras series $(1,6 \%$ vs $2,3 \%$ - 
Tabla 2. Variables intraoperatorias (Grupo con TRR vs Grupo sin TRR)

\begin{tabular}{|lccl|}
\hline Variable & $\begin{array}{c}\text { Grupo con TRR } \\
\mathbf{n = 1 5}\end{array}$ & $\begin{array}{c}\text { Grupo } \\
\text { sin TRR } \\
\mathbf{n}=\mathbf{5 7}\end{array}$ & Comparación \\
\hline Tiempo CEC (min) & $193 \pm 81,2$ & $135 \pm 71,4$ & $\mathrm{p}<0,009$ \\
\hline Clampeo aórtico $(\mathrm{min})$ & $69 \pm 44,5$ & $42 \pm 33,8$ & $\mathrm{p}<0,05$ \\
\hline Temperatura CEC $\left({ }^{\circ} \mathrm{C}\right)$ & $23 \pm 5,2$ & $26,0 \pm 4,7$ & $\mathrm{p}<0,01$ \\
\hline Paro circulatorio & $46,7 \%$ & $12,3 \%$ & OR $6,25($ IC $95 \%: 1,73-22,6)$ \\
\hline Incidentes & $93,3 \%$ & $40,4 \%$ & OR 20,7 (IC 95\%: $2,5-168,4)$ \\
\hline
\end{tabular}

TRR: terapia de reemplazo renal. CEC: circulación extracorpórea.

Tabla 3 Variables post-operatorias (Grupo con TRR vs Grupo sin TRR)

\begin{tabular}{|lccl|}
\hline Variable & $\begin{array}{c}\text { Grupo con TRR } \\
\mathbf{n = 1 5}\end{array}$ & $\begin{array}{c}\text { Grupo sin TRR } \\
\mathbf{n = 5 7}\end{array}$ & Comparación \\
\hline Días en UCl & $20 \pm 16,8$ & $9,3 \pm 5,9$ & $\mathrm{p}<0,009$ \\
\hline Número de DVA & $4,3 \pm 0,6$ & $2,3 \pm 1,3$ & $\mathrm{p}<0,001$ \\
\hline Menor recuento plaquetas $\left(\mathrm{x} \mathrm{mm} \mathrm{mm}^{3}\right)$ & $44.785 \pm 24.934$ & $139.358 \pm 87.830$ & $\mathrm{p}<0,001$ \\
\hline PVC mayor (mmHg) & $23 \pm 8,8$ & $14 \pm 4,9$ & $\mathrm{p}<0,001$ \\
\hline Ácido láctico más alto $(\mathrm{mmol} / \mathrm{L})$ & $10,8 \pm 7,2$ & $4,3 \pm 5,0$ & $\mathrm{p}<0,001$ \\
\hline Hipotensión grave & $86,7 \%$ & $8,8 \%$ & OR 67,6 (IC 95\%: $11,8-388,6)$ \\
\hline Hipodébito persistente & $60 \%$ & $3,5 \%$ & OR 41,3 (IC 95\%: $7,2-237)$ \\
\hline Presencia de CID & $60 \%$ & $17,5 \%$ & OR 7,05 (IC 95\%: $2,0-24,3)$ \\
\hline Mortalidad & $40 \%$ & $7 \%$ & OR 8,83 (IC 95\%: $2,1-37,6)$ \\
\hline
\end{tabular}

TRR: terapia de reemplazo renal. DVA: drogas vasoactivas. PVC: presión venosa central. CID: coagulación intravascular.

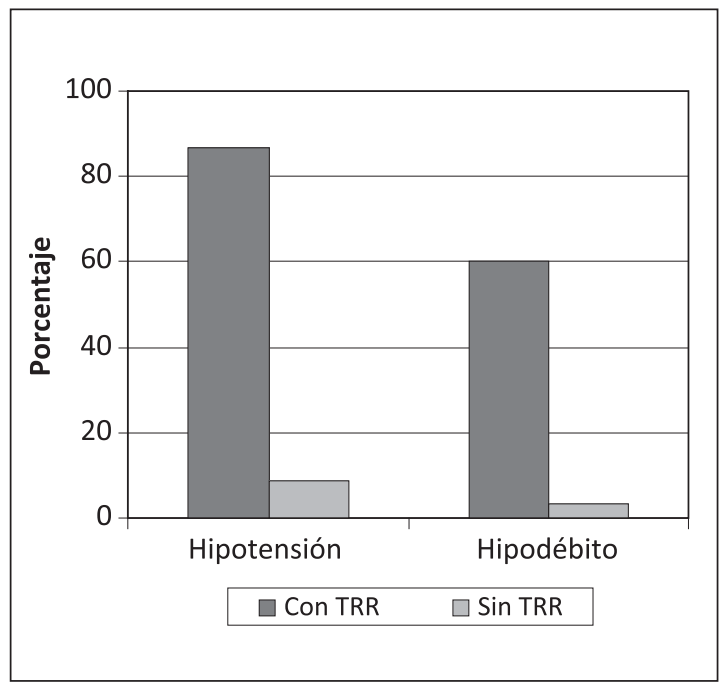

Figura 1. Los factores de riesgo de requerir TRR fueron la hipotensión grave (OR 36,0 [IC 95\%: 5,5-235,6]) y el hipodébito persistente (OR 14,11 [IC 95\%: 1,47-135,3]) en el análisis multivariado.

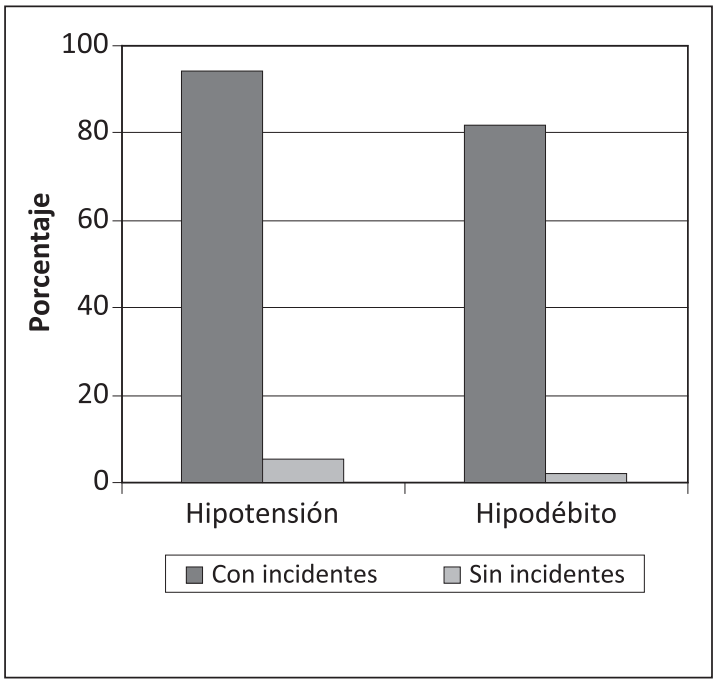

Figura 2. La presencia de incidentes intraoperatorios fue un factor de riesgo para desarrollar hipotensión grave (OR 28,9 [IC 95\%: 3,6-233,9]) e hipodébito persistente (OR 5,3 [IC 95\%: 1,1-26,6]) durante la evolución post-quirúrgica. 
$14,2 \%)^{3,4,8}$. Esta diferencia puede deberse al uso de criterios de inicio de TRR más estrictos o al análisis de pacientes de distinta gravedad o grupos etáreos. Desde enero de 2005, en nuestro hospital se emplea ultrafiltración (MUF: modified ultrafiltration) al final de la CEC en la mayoría de los pacientes, lo cual facilita el manejo de volúmenes durante el post-operatorio y podría reducir la necesidad de TRR por hipervolemia. En otras series no se menciona si se realizó esta terapia. Otro elemento a considerar en nuestros resultados es un potencial subregistro de pacientes, ya que el grupo estudiado fue obtenido en base a la revisión de todas las fichas disponibles de los menores de 18 años sometidos a reparación quirúrgica de cardiopatías congénitas con CEC y a los registros de la UCIP, que pudieran estar incompletos.

La CEC gatilla una importante reacción inflamatoria, con aumento de mediadores que favorecen el leak capilar y, por ende, la hipovolemia y la hipoperfusión renal ${ }^{4}$. Estos factores, al igual que el hipodébito secundario a la falla cardíaca, pueden contribuir a la isquemia renal y derivar en necrosis tubular aguda ${ }^{5}$, llevando finalmente a la falla renal y a la necesidad de instaurar una terapia de reemplazo. Dado que los pacientes cardiópatas toleran mal la sobrecarga de líquidos secundaria a la IRA y que por su gravedad requieren aportes elevados de volumen durante el postoperatorio ${ }^{2}$, en todas las series, incluida la nuestra, las principales indicaciones de TRR son la hipervolemia y/o la oligoanuria ${ }^{6,8,10,14-16}$.

Se han descrito como factores de riesgo de requerir TRR (análisis multivariado) el tiempo de CEC prolongado, el uso de paro cardiocirculatorio y el síndrome de hipodébito ${ }^{10}$. $\mathrm{Al}$ ajustar las variables según la complejidad de las cirugías (de acuerdo a la clasificación de RACHS-1: Risk adjusted classification for congenital heart surgery) ${ }^{17}$, en el análisis multivariado aparecen como factores de riesgo significativos la menor edad y el tiempo de CEC > $90 \mathrm{~min}^{6}$. En nuestra serie, hubo variables tanto intraoperatorias como post-operatorias asociadas a un mayor riesgo de requerir TRR. Las primeras fueron el tiempo de CEC y de clampeo aórtico más prolongado, el uso de paro circulatorio y la presencia de inciden- tes intraoperatorios. Dentro de las segundas, destacan la hipotensión y el hipodébito persistente, que fueron los únicos factores de riesgo significativos en el análisis multivariado, hallazgo que concuerda con lo descrito previamente $^{10}$. El resto de las variables que alcanzan significancia en el análisis univariado reflejan la gravedad de los pacientes durante su evolución post-quirúrgica. El requerimiento de TRR se asoció también a mayor mortalidad y estadía en UCIP más prolongada, del mismo modo como ha sido descrito en otros estudios ${ }^{6}$.

$\mathrm{Al}$ analizar los factores asociados a un mayor riesgo de hipotensión o hipodébito graves, el único significativo fue la presencia de incidentes intraoperatorios. En nuestra serie, éstos fueron graves, causando alteraciones hemodinámicas importantes o necesidad de entrar nuevamente en CEC en varios pacientes. Podría decirse, por lo tanto, que gran parte del pronóstico de estos pacientes está dado por los eventos de la cirugía misma.

La duración de la TRR fue muy variable, al igual que lo reportado en otros estudios ${ }^{4,10}$. Chan y colaboradores encontraron como factores de riesgo de requerir TRR más prolongada una menor edad, una peor función renal preoperatoria y algunas variables postoperatorias como síndrome de hipodébito, hipertensión pulmonar y requerimientos de oxígeno eleva$\operatorname{dos}^{10}$. De nuestros pacientes, $60 \%$ presentaron hipodébito persistente (similar a otras series) ${ }^{10}$, lo cual no se relacionó con una mayor duración de la terapia.

La TRR inicial en todos nuestros pacientes fue $\mathrm{PD}$, al igual que en la mayoría de las series reportadas, ya que es técnicamente poco engorrosa y produce escaso deterioro hemodinámi$\mathrm{Co}^{4}$. La HFVVC, utilizada en un paciente por falla del primer método, si bien puede ser más efectiva en la remoción de volumen, requiere anticoagulación. Además, en muchos casos resulta difícil obtener los accesos vasculares, especialmente en neonatos ${ }^{4}$. Al elegir la TRR inicial, se deben evaluar, caso a caso, los riesgos y beneficios de ambas técnicas, especialmente la experiencia del equipo tratante con cada una de ellas.

Diez de nuestros pacientes (66\%) presentaron complicaciones. Tanto la incidencia como 
el tipo de complicaciones coinciden con lo descrito en otras series (21-74\%) $)^{4,7,10,15,18}$. A diferencia de otros reportes, en nuestros niños se observaron alteraciones hemodinámicas y ventilatorias atribuibles directamente al inicio de la PD por el consiguiente aumento de la presión abdominal. En dos casos, las complicaciones llevaron al cese de la terapia.

La mortalidad en nuestro grupo fue de $40 \%$, lo que está dentro de los rangos reportados por otros autores $(20-80 \%)^{3,4,8,10,15,16}$. Ninguno de los pacientes, tanto en nuestra serie como en las otras ${ }^{4,8,10}$, falleció por una causa directamente renal, si bien la falla renal con la consiguiente sobrecarga de volumen y las consecuencias de la TRR, contribuyen al deterioro hemodinámico y a la falla cardíaca en estos pacientes. La IRA claramente empeora el pronóstico de sobrevida dado que la falla de un órgano deteriora la evolución de otros. Al revisar las distintas series, se observa una disminución de la mortalidad en los pacientes que requieren TRR a lo largo de los últimos 25 años $^{6}$. Como ya se mencionó anteriormente, la introducción de nuevas técnicas quirúrgicas y métodos en el manejo post-operatorio puede haber incidido en esta disminución. Los estudios con menor porcentaje de mortalidad en general incluyen pacientes de menor gravedad o uso de TRR profiláctica desde el inicio del post-operatorio ${ }^{18,19}$.

Desde el punto de vista nefrológico, el pronóstico de los pacientes que sobreviven en general es bueno ${ }^{4,10,18}$. Sin embargo, en nuestra serie cuatro pacientes fueron dados de alta con un clearence de creatinina menor al esperado para la edad. Ahora bien, no contamos con un seguimiento a largo plazo (rango de días hospitalizados: 11 a 20 días). Sería interesante realizar un seguimiento prolongado para evaluar las secuelas de la IRA.

Nuestro estudio tiene algunas limitaciones, ya que es retrospectivo, por lo que no se cuenta con algunos datos que sería interesante evaluar, como por ejemplo la función renal preoperatoria en todos los pacientes, ni hay un protocolo único de tratamiento, sino que depende del equipo médico tratante. Además no hubo acceso a todas las fichas de la muestra aleatoria de pacientes sin TRR. La realización de estudios prospectivos contribuiría a aclarar mejor los factores de riesgo de requerir TRR.

En conclusión, en nuestro estudio la cirugía cardíaca con CEC se asocia a una incidencia de TRR de 1,6\%. La hipotensión grave y el hipodébito persistente aparecen como los principales factores de riesgo de falla renal $\mathrm{y}$ necesidad de terapia de reemplazo. A su vez, la hipotensión y el hipodébito persistentes se relacionan con la presencia de incidentes intraoperatorios. Destacamos la importancia de monitorizar estrictamente la función renal en pacientes con incidentes importantes durante la cirugía, así como de realizar un manejo agresivo de la hipotensión y el hipodébito. Se recomienda considerar el rol del inicio precoz de la TRR durante el post-operatorio inmediato.

\section{Referencias}

1.- Bailey $D$, Phan $V$, Litalien $C$, et al: Risk factors of acute renal failure in critically ill children: A prospective descriptive epidemiological study. Pediatr Crit Care Med 2007; 8: 29-35.

2.- Medina A, López-Herce J, López Y, et al: Insuficiencia renal aguda en niños críticamente enfermos. Estudio preliminar. An Pediatr (Barc) 2004; 61: 509-14.

3.- Cabas L, Ramón F, Kling JC, et al: Disfunción renal en postoperatorio de cirugía cardíaca pediátrica con circulación extracorpórea. Rev Col Anest 2005; 33: 85-91.

4.- Kist-van Holte tot Echten JE, Goedvolk CA, Doornaar $M B M E$, et al: Acute renal insufficiency and renal replacement therapy after pediatric cardiopulmonary bypass surgery. Pediatr Cardiol 2001; 22: 321-6.

5.- Baskin E, Saygili A, Harmanci K, et al: Acute renal failure and mortality after open-heart surgery in infants. Ren Fail 2005; 27: 557-60.

6.- Pedersen KR, Povlsen JV, Christensen S, et al: Risk factors for acute renal failure requiring dialysis after surgery for congenital heart disease in children. Acta Anaesthesiol Scand 2007; 51: 1344-9.

7.- Vogel A, Díaz M, Aglony M, Cavagnaro F, Rodríguez JI, Lagomarsino E: Peritoneodiálisis en pacientes pediátricos postoperados de cirugía cardíaca. Congreso Conjunto de Nefrología, Hipertensión y trasplante, 2, 3 y 4 de Octubre, 2003. La Serena-Chile.

8.- Boigner H, Brannath W, Hermon M, et al: Predictors of mortality at initiation of peritoneal dialysis in children after cardiac surgery. Ann Thorac Surg 2004; 77: 61-5. 
9.- Jander A, Tkaczyk M, Pagowska-Klimek I, et al: Continuous veno-venous hemodiafiltration in children after cardiac surgery. Eur J Cardiothorac Surg 2007; 31: 1022-8.

10.- Chan $K$, Ip P, Chiu C, Cheung Y: Peritoneal dialysis after surgery for congenital heart disease in infants and young children. Ann Thorac Surg 2003; 76: 1443-9.

11.- Haque IU, Zaritsky AL: Analysis of the evidence for the lower limit of systolic and mean arterial pressure in children. Pediatr Crit Care Med 2007; 8: 138-44.

12.- Schwartz GJ, Brion Lp, Spitzer A: The use of plasma creatinine concentration for estimating glomerular filtration rate in infants, children, and adolescents. Pediatr Clin North Am 1987; 34: 571-90.

13.- Fons J, Peris A, Hervás A, et al: Estudio de la función renal en el recién nacido. En Delgado A, editor: Protocolos diagnósticos y terapéuticos de NefrologíaUrología Pediátrica 2000. Disponible en: http://www. aeped.es/protocolos/nefro/index.htm. Última visita 18-02-2009.

14.- Alkan T, Akçevin A, Türkoglu H, et al: Postoperative prophylactic peritoneal dialysis in neonates and infants after complex congenital cardiac surgery. ASAIO Journal 2006; 52: 693-7.

15.- Pedersen KR, Hjortdal VE, Christensen S, et al: Clinical outcome in children with acute renal failure treated with peritoneal dialysis after surgery for congenital heart disease. Kidney Int 2008; 73: S81-6.

16.- Romão JE, Fuzissima MG, Vidonho AF, et al: Outcome of acute renal failure associated with cardiac surgery in infants. Arq Bras Cardiol 2000; 75: 318-21.

17.- Jenkins KJ, Gauvreau K, Newburger JW, Spray TL, Moller JH, Jezzoni LI: Consensus-based method for risk adjustment for surgery for congenital heart disease. J Thorac Cardiovasc Surg 2002; 123: 110-8.

18.- Dittrich S, Dähnert I, Vogel M, et al: Peritoneal dialysis after infant open heart surgery: Observation in 27 pacientes. Ann Thorac Surg 1999; 68: 160-3.

19.- Sorof JM, Stromberg D, Brewer ED, Feltes TF, Fraser $C D J r$ : Early initiation of peritoneal dialysis after surgical repair of congenital heart disease. Pediatr Nephrol 1999; 13: 641-5. 